\title{
Prototipação de Aplicativos como Método de Aprendizagem na Informática em Saúde: Um Relato de Experiência
}

\author{
Lidiany C. Santos ${ }^{1,3}$, Danilo F. Neves ${ }^{2,3}$, Hélio C. de Moura Filho ${ }^{3}$, \\ Fabrício dos Santos Menezes ${ }^{1}$, Luis Felipe S. da Silva ${ }^{1}$ \\ ${ }^{1}$ Departamento de Educação em Saúde - Universidade Federal de Sergipe (UFS) \\ Lagarto - SE - Brasil \\ ${ }^{2}$ Programa de Pós-Graduação em Ciência da Computação - UFS \\ São Cristóvão - SE - Brasil \\ ${ }^{3}$ Serviço de Tecnologia da Informação - UFS \\ Lagarto - SE - Brasil \\ \{lidianycs, danilo.neves, helio.cardoso, fabriciomenezes, luisfelipegamboa\}@ufs.br
}

\begin{abstract}
Informatics has been assuming a relevant role in the health field, evidencing the need for trained professionals to use digital technologies. This work presents an experience report on the teaching of Applied Informatics for Health. It was adopted an active methodology and prototyping of mobile apps was used as a learning method. Positive results indicate that the experience can be continued and improved upon further study. The methodology adopted and the results achieved are described in this work, in order to contribute to the replication and improvement of the experience.
\end{abstract}

Resumo. A informática vem assumindo um papel relevante no campo da saúde, evidenciando a necessidade de profissionais capacitados para o uso de tecnologias digitais. Este trabalho apresenta um relato de experiência sobre o ensino da Informática Aplicada à Saúde. Adotou-se uma metodologia ativa e a prototipação de aplicativos móveis foi utilizada como método de aprendizagem. Resultados positivos indicam que a experiência pode ser continuada e melhorada após um estudo mais aprofundado. A metodologia adotada e os resultados alcançados estão descritos neste trabalho, a fim de contribuir para a replicação e melhoria da experiência.

\section{Introdução}

Avanços da computação e das Tecnologias Digitais da Informação e Comunicação (TDIC) tem impulsionado o desenvolvimento da informática aplicada à saúde. Sistemas de informação em saúde tornaram-se fundamentais para atividades da área, como por exemplo, a prática de vigilância, monitoramento de agravos, indicadores de morbidade e mortalidade, suporte à pequisa, à gestão e apoio à tomada de decisão [Cardoso et al. 2008, Nichiata et al. 2003]. O interesse por esse setor tornou-se evidente nos últimos anos com a popularização das maratonas de desenvolvimento que propõem desafios de informática voltados para o campo da saúde. Porém, apesar da ampliação expressiva na realização desses eventos, ainda é observada uma pequena participação de profissionais de Saúde [Guizardi et al. 2018]. 
VII Congresso Brasileiro de Informática na Educação (CBIE 2018)

Anais do XXIV Workshop de Informática na Escola (WIE 2018)

O desenvolvimento da informática e das tecnologias promoveram mudanças nas relações entre os seres humanos e desses com o meio, tornando imprescindível que profissionais atualizados consigam discutir e desenvolver competências para a utilização de recursos computacionais na sua área [Cardoso et al. 2008]. O emprego de tecnologias e dispositivos móveis demonstra ser uma boa estratégia para a promoção da educação em saúde, contribuindo para o progresso do setor [Farias et al. 2015]. Com a utilização intensiva das TDIC na saúde, é essencial que estudantes e profissionais conheçam o potencial e dominem as tecnologias para adotá-las em sua rotina. Contudo, a oferta da disciplina informática em saúde nos cursos de graduação ainda encontra dificuldades, tanto por falta de profissionais capacitados para o ensino, quanto por falta de infraestrutura adequada [Santos et al. 2017].

Além do desenvolvimento tecnológico, a educação em saúde vem sendo transformada também pela adoção de metodologias ativas, estimulando a autonomia, a participação e proatividade dos discentes. Investimentos e experiências neste setor vem modificando a formação em saúde, oferecendo um panorama híbrido, no qual a teoria e prática se complementam e os estudantes são responsáveis pela sua própria formação [Aguiar et al. 2014, Pereira et al. 2017].

Este artigo relata a experiência da prática pedagógica da informática em saúde por meio da disciplina optativa de Informática Aplicada à Saúde, ofertada pelo Departamento de Educação em Saúde da Universidade Federal de Sergipe (UFS) no Campus Lagarto. O objetivo do presente relato é apontar algumas possibilidades de prática docente, por meio da Aprendizagem Baseada em Problemas (ABP) e da utilização das TDIC no ensino de informática em saúde. Este trabalho foi motivado por entender-se que a metodologia escolhida pode enriquecer as oportunidades educacionais e fomentar a interdisciplinaridade inerente à disciplina.

Foi proposto aos estudantes, o desenvolvimento de um protótipo de aplicativo para dispositivos móveis com aplicação na área da saúde. Os participantes foram estimulados a pensar em problemas reais e identificar oportunidades para criação de um protótipo com aplicação em suas áreas de estudo. A estratégia adotada mostrou-se desafiadora para os estudantes. Entretanto, a apresentação de outros aplicativos desenvolvidos por profissionais de saúde, motivou os estudantes a assumirem o papel de projetistas e desenvolvedores.

Nas seções subsequentes é apresentada uma descrição mais detalhada das ações realizadas na disciplina. Na seção 2 é descrita a metodologia empregada. Na seção 3 são descritos os resultados alcançados e por fim, na seção 4 são discutidas as conclusões e possibilidades de trabalhos futuros.

\section{Metodologia}

A adoção de metodologias ativas no ensino superior em saúde tem como objetivo formar profissionais com habilidades e competências além do domínio técnico-científico [Pereira et al. 2017]. O ensino centrado no aluno busca incentivar a capacidade de criação, planejamento e de solucionar problemas [Barron et al. 1998]. A estrutura curricular dos cursos de graduação ofertados pela UFS no Campus de Ciências da Saúde em Lagarto/SE, prevê a adoção de metodologias ativas no processo de ensino-aprendizagem [Aguiar et al. 2014]. Para a oferta da disciplina optativa de Informática Aplicada à Saúde, 
VII Congresso Brasileiro de Informática na Educação (CBIE 2018)

Anais do XXIV Workshop de Informática na Escola (WIE 2018)

optou-se pela adoção da metodologia ativa denominada Aprendizagem Baseada em Projetos (ABP). Esta metodologia é uma abordagem sistêmica, na qual se busca o desenvolvimento da capacidade criativa dos discentes e de habilidades e competências por meio do raciocínio e da comunicação entre os pares, adotando-se um processo de investigação de questões complexas, tarefas e produtos planejados para uma aprendizagem eficiente e eficaz [Barron et al. 1998, Masson et al. 2012, Campos et al. 2016]. Na ABP, os estudantes se tornam agentes na construção de conhecimentos e competências, rompendo-se com a forma rígida e pré-estabelecida do desenvolvimento dos conteúdos e permitindose que estes sejam incorporados pelos discentes ao longo do desenvolvimento do projeto [Masson et al. 2012].

A disciplina foi ofertada no período 2017.2, entre dezembro de 2017 e abril de 2018, para os discentes da graduação. Participaram da turma, 21 estudantes dos cursos de Enfermagem, Farmácia, Fisioterapia, Fonoaudiologia, Nutrição, Medicina e Terapia Ocupacional. A docente que orientou os projetos dos discentes possui Bacharelado em Engenharia de Computação e Mestrado em Ciência da Computação, atuando na área de Engenharia de Software e Informática em Saúde.

Como parte da estratégia da ABP, foi apresentado aos discentes um projetodesafio, no qual o papel docente consistiu na mediação e articulação do processo de ensino-aprendizagem. O projeto compreendeu o desenvolvimento de um protótipo de aplicativo para dispositivos móveis com aplicação na área da saúde ou educação em saúde. No desenvolvimento de aplicativos móveis, a interface de usuário é um dos fatores importantes para o sucesso das aplicações, por isso torna-se importante adotar uma metodologia de desenvolvimento que permita avaliar o software o mais cedo possível. A prototipação pode auxiliar neste sentido, uma vez que auxilia na modelagem e documentação das aplicações, bem como no levantamento de requisitos, possibilita a detecção de falhas e que as decisões tomadas em estágios iniciais de desenvolvimento sejam realizadas em conjunto com os usuários [de Oliveira et al. 2012]. O processo de prototipação foi escolhido pois parte de cenários concretos de uso, passando por sucessivos refinamentos até se obter uma visão abrangente e detalhada do funcionamento do sistema em desenvolvimento [Oliveira et al. 2005]. Além disso, optou-se pela prototipação devido aos estudantes não estarem familiarizados com conceitos de programação e desenvolvimento de software e também pela facilidade de utilização das ferramentas disponíveis para prototipação, sendo possível desenvolver os projetos sem a necessidade de implementação dos programas.

Após introduzir os conceitos como algoritmos e prototipação, também foram apresentados alguns casos de sucesso e exemplos de aplicativos na área da saúde, com o objetivo de motivá-los. Em seguida, os estudantes foram separados em grupos multidisciplinares com 4 a 6 participantes e estimulados a identificar soluções para problemas reais em suas respectivas áreas de estudo. Então, os estudantes começaram a pensar sobre o projeto, pesquisar informações sobre o que desejavam explorar, discutir as ideias entre o grupo e a turma sobre a aplicação. As atividades realizadas tiveram como objetivo favorecer a aprendizagem colaborativa e participativa, bem como promover discussões acerca dos benefícios providos pela tecnologia, destacando a importância da sua aplicação na saúde e na sociedade como um todo. A Figura 1 apresenta as principais atividades realizadas ao longo da disciplina.

A primeira atividade consistiu na apresentação de um quadro geral sobre a in- 


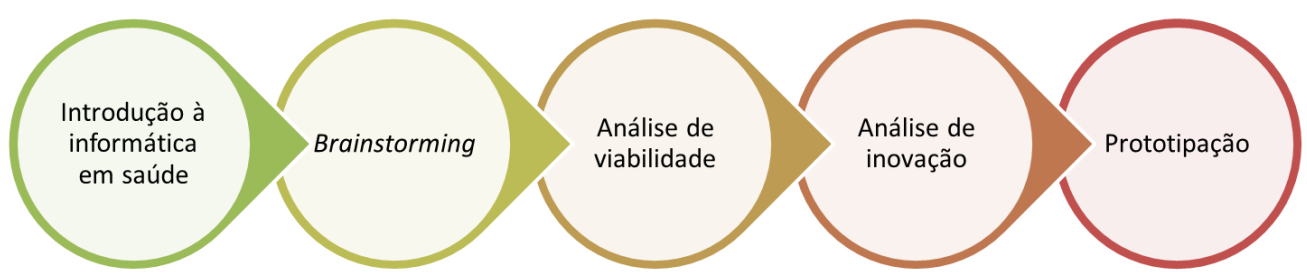

Figura 1. Atividades realizadas

formática na saúde, sua aplicação e histórico, visando introduzir a temática da disciplina para os discentes. Em seguida, foram apresentados alguns exemplos de aplicativos desenvolvidos por equipes multidisciplinares com aplicação na área da saúde, como os aplicativos públicos e gratuitos desenvolvidos pelo núcleo de Telessaúde da Universidade Federal do Rio Grande do Sul (UFRGS) ${ }^{1}$. Foi solicitado aos estudantes que realizassem a instalação e teste de alguns aplicativos, ao mesmo tempo em que foram estimulados a pensar na possibilidade de desenvolver seu próprio protótipo. Essas atividades foram realizadas para familiarizar os estudantes com programas desenvolvidos e disponibilizados para o público e também exemplificar os tipos de aplicações que poderiam ser desenvolvidas ao longo da disciplina.

Dentre as atividades, foi promovido um brainstorming, que consiste em uma técnica para estimular a criatividade e inovação, contribuindo para a geração de ideias em conjunto. Os estudantes formaram as suas equipes e foram estimulados a pensar no tipo de aplicativo que iriam propor. Essa técnica adotada, promoveu uma reunião de estudantes de diferentes cursos, colocando-os em um papel diferente do que estão acostumados, ou seja, saindo do papel de consumidores da tecnologia para o papel de projetistas. Por meio desta estratégia, um dos objetivos foi estimular a proposição de soluções inovadoras apresentadas pelos discentes.

Após essa etapa, foi realizada uma análise de viabilidade das soluções apresentadas. Foi analisado se as ideias propostas seriam factíveis com as tecnologias e recursos disponíveis atualmente.

Também foi avaliada a inovação das propostas. Foi solicitado aos discentes que utilizassem ferramentas de busca para verificar trabalhos anteriormente desenvolvidos ou projetos relacionados. Caso fossem encontrados trabalhos relacionados, as equipes teriam que oferecer um diferencial em relação à estes.

Após estas etapas, foram apresentados aos discentes o conceito de prototipação e algumas ferramentas para o desenvolvimento de protótipos de aplicativos como Wireframe ${ }^{2}$, Gravit designer ${ }^{3}$ e Ninja Mock $4^{4}$. Foi demonstrado aos estudantes como fazer uso dessas ferramentas e facultada a eles a escolha de qual ferramenta desejariam adotar. Durante esta etapa, os estudantes trabalharam na interface da solução idealizada pelas equipes. Realizou-se ainda, a apresentação para os discentes da definição de algoritmos e foi solicitado que eles descrevessem e planejassem as funcionalidades dos aplicativos

\footnotetext{
${ }_{1}^{1}$ www.ufrgs.br/telessauders/desenvolvimento/aplicativos/

2 WWW.wireframe.cC

WwW.designer.io

WwW.ninjamock.com
} 
VII Congresso Brasileiro de Informática na Educação (CBIE 2018)

Anais do XXIV Workshop de Informática na Escola (WIE 2018)

prototipados.

Como requisitos para aprovação dos protótipos definidos pelos grupos, foi definido que a solução apresentada deveria ser inovadora, ter aplicação na área da saúde ou educação em saúde e ser factível de implementar.

A cada encontro da disciplina, com carga horária semanal de quatro horas, foram reservadas duas horas semanais para que os estudantes se reunissem e discutissem as ideias do projeto. No sexto encontro, foi definida uma apresentação preliminar, na qual os grupos deveriam ter definido qual seria o protótipo, a motivação para o seu desenvolvimento e o público-alvo. No décimo quinto e último encontro da disciplina, foi realizada a apresentação final, na qual foram apresentados os protótipos finalizados e discutidas as funcionalidades de cada um.

\section{Resultados}

Como produto final das atividades, cada equipe apresentou um protótipo de aplicação de média fidelidade para dispositivos móveis. As equipes apresentaram os seguintes protótipos:

1. Tempo ao Sol (Figura 2) - um aplicativo para orientar os usuários quanto à necessidade e aos cuidados com a exposição solar, visando a melhoria de absorção de vitamina D;

2. Crescer Feliz (Figura 3) - um aplicativo para auxiliar pais e mães no acompanhamento das necessidades do bebê e fornecer orientações precisas de saúde, esclarecendo mitos e boatos que permeiam a puericultura, além de auxiliar os profissionais de saúde na obtenção de dados relevantes;

3. Meu Gasto Energético (Figura 4) - um aplicativo que realiza o cálculo do gasto de energia total diário por individuo, através do acompanhamento e registro diário do consumo de alimentos, oferecendo orientações acerca de uma alimentação saudável e equilibrada;

4. Libras em Saúde (Figura 5) - um aplicativo para auxiliar no ensino-aprendizagem da Língua Brasileira de Sinais (Libras), voltado para estudantes e profissionais de saúde.

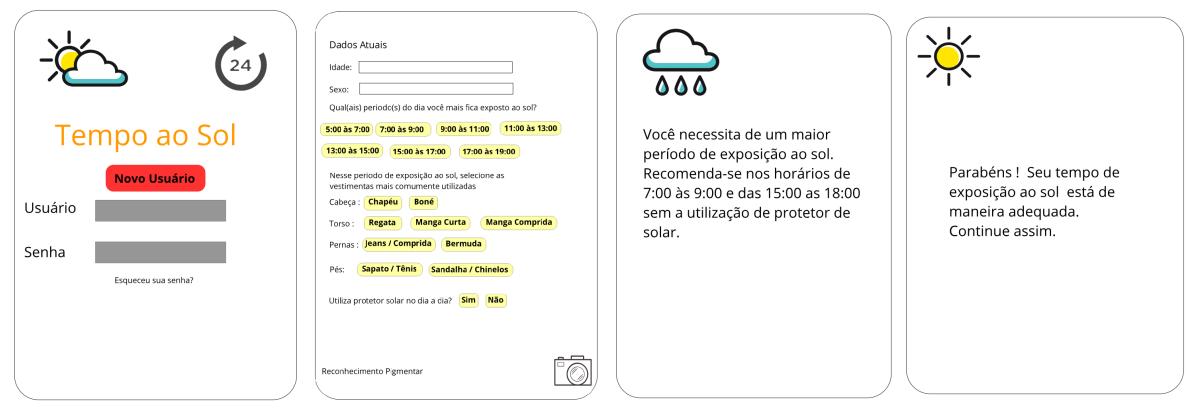

Figura 2. Protótipo de aplicativo Tempo ao Sol

Foi observada desde o início, a multidisciplinaridade das soluções propostas pelas equipes. A equipe que propôs o desenvolvimento da aplicação Tempo ao Sol, apresentado na Figura 2, era composta por 2 estudantes do curso de Medicina, 1 estudante do curso de Farmácia e 1 estudante de Terapia Ocupacional. Foi observada a preocupação dos 
VII Congresso Brasileiro de Informática na Educação (CBIE 2018)

Anais do XXIV Workshop de Informática na Escola (WIE 2018)
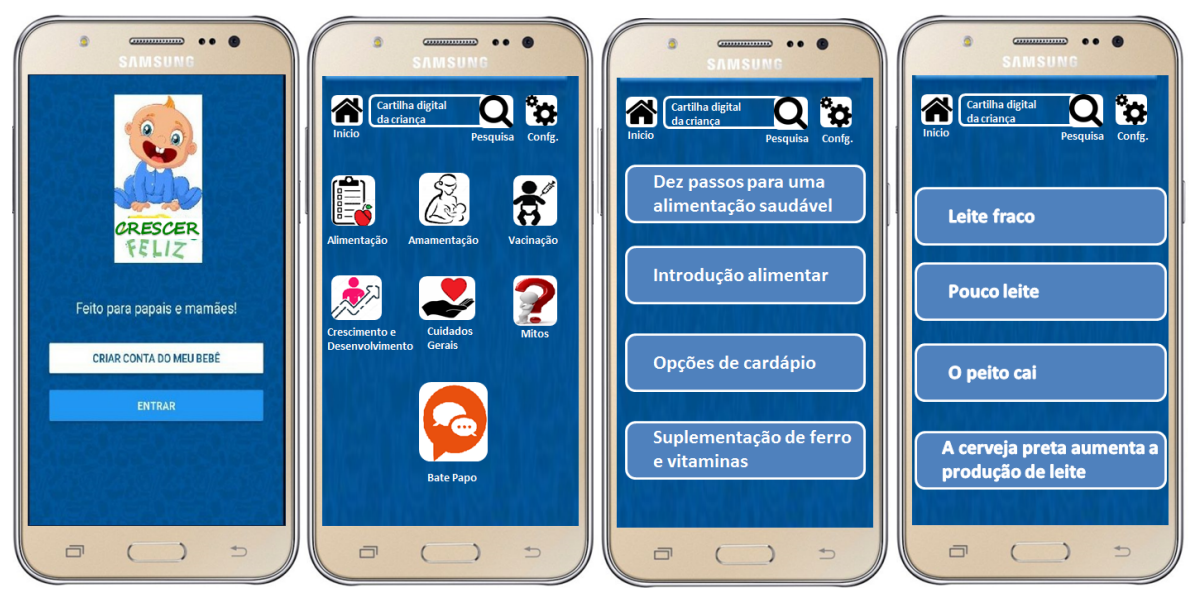

Figura 3. Protótipo de aplicativo Crescer Feliz
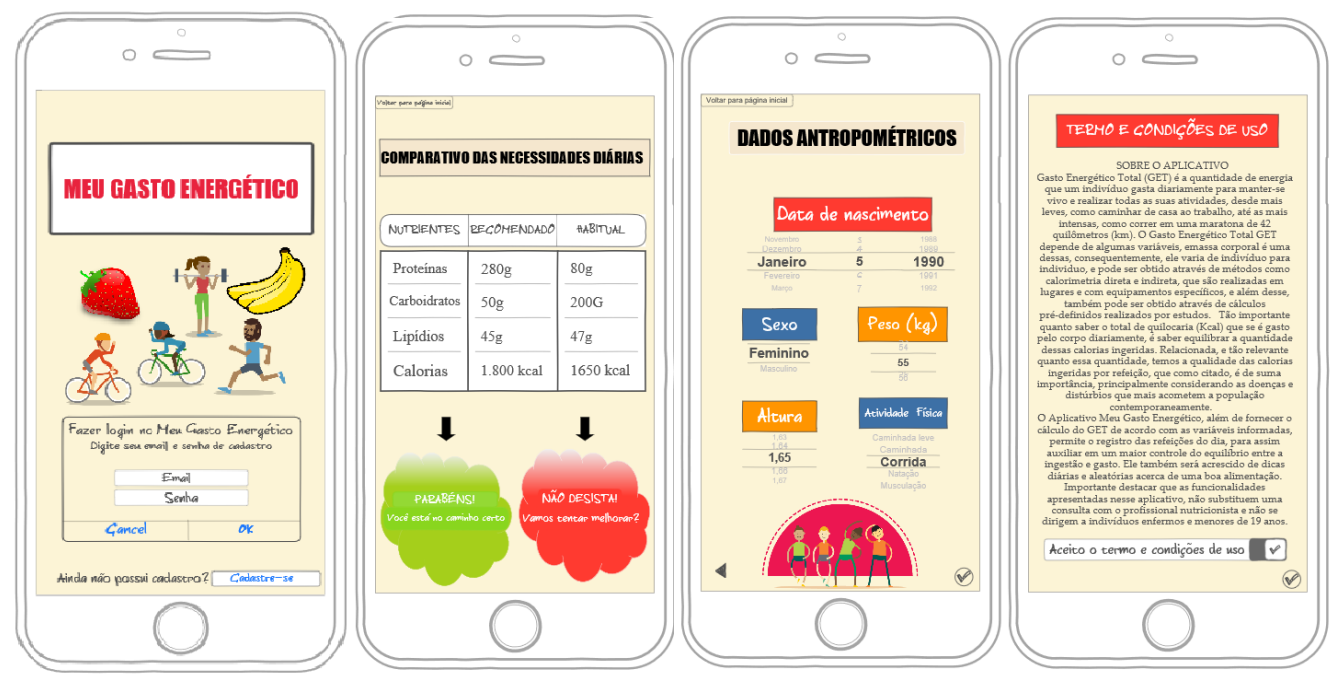

Figura 4. Protótipo de aplicativo Meu gasto Energético

integrantes em orientar os usuários do app quanto à necessidade da exposição solar para melhor absorção da vitamina $\mathrm{D}$, bem como quanto à necessidade do uso de proteção solar em determinados horários.

A equipe que propôs o aplicativo Crescer Feliz, apresentado na Figura 3, foi integrada por discentes dos cursos de Medicina, Enfermagem e Nutrição. Neste grupo, houve uma preocupação com a coleta de dados essenciais para o acompanhamento do crescimento infantil e com as dúvidas e mitos bastante difundidos acerca da puericultura.

O único grupo que não apresentou uma composição multidisciplinar foi o responsável pela aplicação Meu Gasto Energético, apresentado na Figura 4 . Este grupo propôs uma solução aplicada à Nutrição, com ênfase na alimentação saudável, o que se mostrou coerente, uma vez que todas as discentes integrantes da equipe eram acadêmicas do referido curso.

A equipe responsável pelo aplicativo Libras em Saúde, apresentado na Figura 5 , tinha como integrantes 3 discentes do curso de Fonoaudiologia, 2 discentes de Nutrição e 1 discente de Fisioterapia. Este grupo salientou que a disciplina de Introdução à Libras 
VII Congresso Brasileiro de Informática na Educação (CBIE 2018)

Anais do XXIV Workshop de Informática na Escola (WIE 2018)
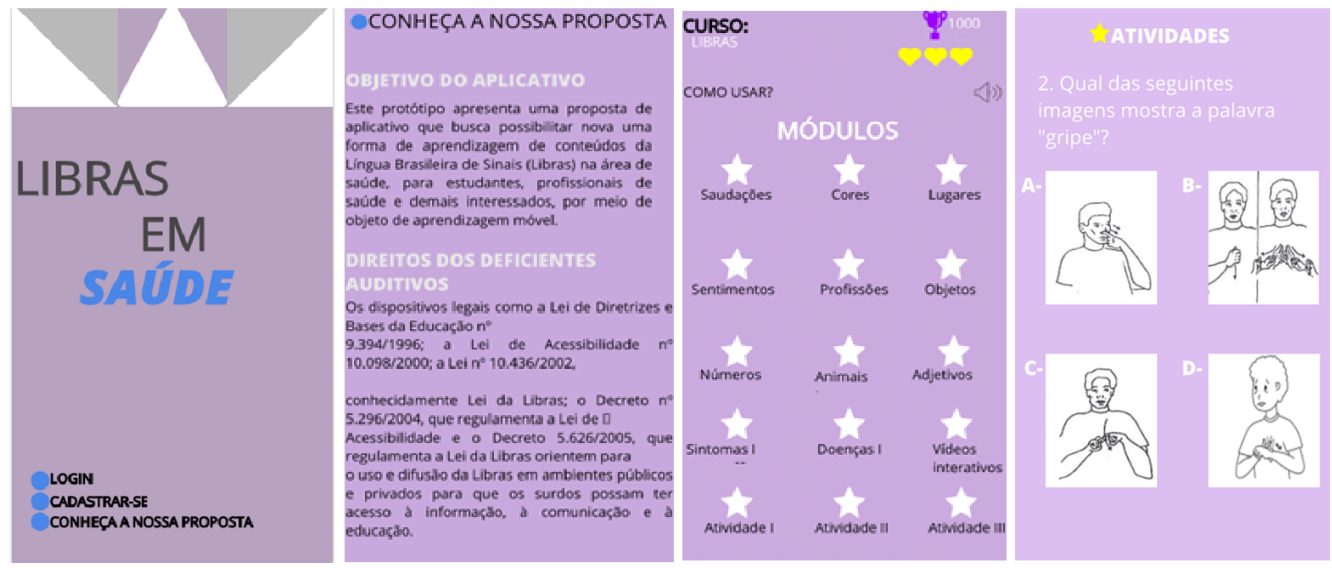

Figura 5. Protótipo de aplicativo Libras em Saúde

não é obrigatória para todos os cursos da saúde do Campus e que a sua oferta pelas universidades também não é obrigatória. Contudo, conforme destacou o grupo, o conhecimento de Libras pelos profissionais de saúde é essencial, verificando-se assim a necessidade de ações para promover o aprendizado da língua entre estudantes e profissionais de saúde. A composição multidisciplinar do grupo favoreceu a apresentação de uma solução voltada para auxiliar profissionais da saúde com formações diversas.

A adoção da ABP mostrou-se um desafio para os estudantes, apesar de já estarem familiarizados com as metodologias ativas. Mesmo fazendo uso de dispositivos móveis e aplicativos constantemente, foi observada uma resistência inicial dos estudantes em assumir o papel de projetistas e desenvolvedores, uma perspectiva nova para a maioria deles. Apenas um dos discentes que participaram da disciplina tinha experiência no desenvolvimento de software por ter cursado uma graduação anterior em Engenharia de Computação, os demais relataram ter conhecimentos básicos de informática. Percebeu-se que a princípio os discentes apresentavam certa insegurança com a responsabilidade de conduzir o projeto e apresentar uma solução. Entretanto, após a apresentação de outros trabalhos na área, os estudantes foram motivados a seguir com o projeto.

Os comentários dos discentes foram positivos em relação a metodologia aplicada. Abaixo são destacadas alguns pontos citados pelos discentes participantes:

"Apresentou conhecimentos e informações que não são vistos nas aulas pertencentes às disciplinas normativas e possibilitou o contato com outras habilidades e áreas."

"Agilidade, inovação e interdisciplinaridade". "Ajuda na compreensão melhor da informática na área da saúde".

Os próprios estudantes apresentaram algumas preocupações e questionamentos acerca dos softwares e aplicativos estudados em sala de aula. Foram discutidos alguns aspectos em relação ao uso da tecnologia como: o uso de aplicativos e da internet não substituírem o atendimento profissional, o sigilo das informações e a segurança dos pacientes. Esses questionamentos se tornaram requisitos aplicados nas soluções projetadas. 
VII Congresso Brasileiro de Informática na Educação (CBIE 2018)

Anais do XXIV Workshop de Informática na Escola (WIE 2018)

\section{Considerações Finais e Trabalhos Futuros}

O impacto da tecnologia na saúde e na educação tornou-se inevitável. A adoção de ferramentas e metodologias para estimular a criatividade, explorando, planejando, pesquisando e ampliando seus horizontes, contribui significativamente para o aprendizado. Outra contribuição significativa, diz respeito ao encorajamento dos discentes a assumirem uma postura proativa e empreendedora, desafiando-os a sair do papel de consumidores das tecnologias para tornarem-se projetistas e inventores.

As atividades realizadas na disciplina tiveram como objetivo despertar o senso crítico dos discentes diante da adoção das tecnologias no setor de saúde, bem como de familiarizá-los com a informatização do setor. Procurou-se ambientar os discentes com o processo de desenvolvimento de software e também com o uso de computadores e dispositivos móveis como ferramentas de trabalho em suas respectivas áreas de atuação. Os discentes foram incentivados a participar ativamente na proposição de soluções de tecnologia da informação e a trabalhar coletivamente com foco na inovação e no desenvolvimento tecnológico, colocando-os em uma nova perspectiva diante das TDIC.

Ao final da disciplina foi possível observar que os discentes mostraram-se satisfeitos com os resultados alcançados. Dentre os 22 estudantes matriculados, houve apenas uma reprovação por parte de uma estudante que não frequentou nenhuma aula do curso. Todos os discentes foram aprovados com médias entre 8 e 10 pontos. Uma sugestão de melhoria para o próximo período apresentada pelos estudantes foi o desenvolvimento funcional dos protótipos, pois gostariam de vê-los em funcionamento. Tendo como base os os resultados obtidos através desta pesquisa, é possível sugerir novas experiências no ensino de informática em saúde, nas quais estudantes e professores consigam interagir positivamente com as TDIC.

Diante dos resultados positivos com a metodologia aplicada nesta experiência, esta deve ser aprimorada para adoção nas próximas turmas. O próximo passo consiste na adoção da plataforma de programação visual App Inventor ${ }^{5}$, desenvolvida pelo Instituto de Tecnologia de Massachusetts (MIT) em parceria com o Google. O App Inventor propõe uma metodologia fácil, simples e intuitiva para o aprendizado e desenvolvimento de aplicativos. Esta ferramenta deverá ser usada pelos discentes para o desenvolvimento de protótipos funcionais. Será incluída também, uma etapa de avaliação dos protótipos desenvolvidos que deverá ser realizada entre as equipes participantes. Pretende-se ainda estudar medidas que possibilitem uma avaliação mais objetiva dos efeitos da metodologia na aprendizagem.

\section{Referências}

[Aguiar et al. 2014] Aguiar, R. G. d., Barbosa, G. R., de Oliveira, A. M. B., Moccellin, A. S., Costa, M. R. d. C. D., and Silveira, N. A. (2014). Implantação de um curso de fisioterapia baseado em metodologias ativas de ensino-aprendizagem. Cadernos de educação, Saúde e fisioterapia, 1(1).

[Barron et al. 1998] Barron, B. J., Schwartz, D. L., Vye, N. J., Moore, A., Petrosino, A., Zech, L., and Bransford, J. D. (1998). Doing with understanding: Lessons from re-

\footnotetext{
5 www.appinventor.mit.edu
} 
VII Congresso Brasileiro de Informática na Educação (CBIE 2018)

Anais do XXIV Workshop de Informática na Escola (WIE 2018)

search on problem-and project-based learning. Journal of the Learning Sciences, 7(34):271-311.

[Campos et al. 2016] Campos, A. d., Machado, G. B., Rados, G. J. V., and Todesco, J. L. (2016). Aprendizagem baseada em projetos: uma experiência em sala de aula para compartilhamento e criação do conhecimento no processo de desenvolvimento de projetos de software. Revista Competência, 9(2):17-35.

[Cardoso et al. 2008] Cardoso, J. P., Rosa, V. A., Lopes, C. R. S., Vilela, A. B. A., Santana, A. S. d., and Silva, S. T. d. (2008). Construção de uma práxis educativa em informática na saúde para ensino de graduação. Ciência \& Saúde Coletiva, 13:283-288.

[de Oliveira et al. 2012] de Oliveira, D. H. D., de Miranda, L. C., de Miranda, E. E. C., and da Silva, L. F. (2012). Prototipação de interfaces de aplicativos para dispositivos móveis: Estado da arte e desafios de ihc. In Proceedings of the 11th Brazilian Symposium on Human Factors in Computing Systems, IHC'12, pages 315-324, Porto Alegre, Brazil, Brazil. Brazilian Computer Society.

[Farias et al. 2015] Farias, A., Pereira, M., Souto, M., and Alencar, R. (2015). Educação em saúde no brasil: uma revisão sobre aprendizagem móvel e desafios na promoção de saúde no brasil. In Anais do Workshop de Informática na Escola, volume 21, page 614.

[Guizardi et al. 2018] Guizardi, F. L., Santos, K. F. d., Lemos, A. S. P., and Severo, F. M. D. (2018). Hackathons in brazil and the challenges they pose to the field of health. Interface-Comunicação, Saúde, Educação, 22(65):447-460.

[Masson et al. 2012] Masson, T. J., Munhoz Jr, A. H., and Castanheira, A. M. P. (2012). Metodologia de ensino: aprendizagem baseada em projetos (pbl). In Congresso Brasileiro de Educação em Engenharia (COBENGE).

[Nichiata et al. 2003] Nichiata, L. Y. I., Takahashi, R. F., Fracolli, L. A., Lins, A. L. d. F. P., et al. (2003). Relato de uma experiência de ensino de enfermagem em saúde coletiva: a informática no ensino de vigilância epidemiológica. Revista da Escola de Enfermagem da USP, 37(3):36-43.

[Oliveira et al. 2005] Oliveira, J. M. P. d., Fernandes, C. T., Ramos, E. S., Jacinto, A., Vilela, F., and Ajukas, D. (2005). Processo de prototipação da interação em sti que utiliza a aprendizagem baseada em problemas como proposta pedagógica. In Brazilian Symposium on Computers in Education (Simpósio Brasileiro de Informática na Educação-SBIE), volume 1, pages 383-393.

[Pereira et al. 2017] Pereira, S., Capelli, J. C. S., Abrahão, A. L., and Anastacio, A. (2017). A experiência do uso da aprendizagem baseada em projetos como metodologia ativa no programa de educação pelo trabalho para a saúde na aprendizagem da prática profissional. DEMETRA: Alimentação, Nutrição \& Saúde, 12(4):881-898.

[Santos et al. 2017] Santos, M. A. d., de Paiva Guimarães, M., and Abe, K. C. (2017). O ensino da disciplina de informática em saúde nos cursos de graduação em enfermagem. EmRede-Revista de Educação a Distância, 4(1):166-173. 